\title{
OPEN NEWTON - COTES QUADRATURE WITH MIDPOINT DERIVATIVE FOR INTEGRATION OF ALGEBRAIC FUNCTIONS
}

\author{
T. Ramachandran ${ }^{1}$, R.Parimala ${ }^{2}$ \\ ${ }^{1}$ Assistant Professor, Department of Mathematics, M.V.Muthiah Government Arts College for Women, \\ Tamil Nadu, India \\ ${ }^{2}$ Research Scholar, PG \&Research Department of Mathematics, Government Arts College (Autonomous), \\ Tamil Nadu, India
}

\begin{abstract}
Many methods are available for approximating the integral to the desired precision in Numerical integration. A new set of numerical integration formula of Open Newton-Cotes Quadrature with Midpoint Derivative type is suggested, which is the modified form of Open Newton-Cotes Quadrature. This new midpoint derivative based formula increase the two order of precision than the classical Open Newton-Cotes formula and also gives more accuracy than the existing formula. Further the error terms are obtained and compared with the existing methods. Finally, the effectiveness of the proposed algorithm is illustrated by means of a numerical example.
\end{abstract}

Key Words: Numerical Integration, Open Newton-Cotes formula, Midpoint Derivative, Numerical Examples.

\section{INTRODUCTION}

Numerical integration is the process of computing the value of a definite integral from a set of numerical values of the integrand. The process of evaluation of integration of a function of a single variable is sometimes called Mechanical Quadrature. The computation of a double integral of a function of two independent variables is called Mechanical Cubature. There are many methods are available for numerical integration [1]. Consider the definite integral

$$
I(f)=\int_{a}^{b} f(x) d x
$$

where the function $f(x)$ is continuous in the closed interval [a,b], so that the integral I(f) exists. An efficient formula is developed for computing approximate value of the integral using only values of the integrand $f(x)$ at points $x \in[a, b]$. To approximate the integral $I(f)$, we integrate exactly piecewise polynomial approximations of $\mathrm{f}(\mathrm{x})$ on the interval $[\mathrm{a}, \mathrm{b}]$. Generally, a quadrature rule has the form,

$$
\int_{\mathrm{a}}^{\mathrm{b}} \mathrm{f}(\mathrm{x}) \mathrm{dx} \approx \sum_{i=0}^{n} w_{i} f\left(x_{i}\right)
$$

Where there $(\mathrm{n}+1)$ distinct points $\mathrm{x}_{0}<\mathrm{x}_{1}<\ldots<\mathrm{x}_{\mathrm{n}}$ and $(\mathrm{n}+1)$ weights $\mathrm{w}_{0}, \mathrm{w}_{1}, \ldots, \mathrm{w}_{\mathrm{n}}$ within the interval [a,b]. The error of approximation is given as,

$$
\mathrm{E}_{\mathrm{n}}[\mathrm{f}]=\int_{\mathrm{a}}^{\mathrm{b}} \mathrm{f}(\mathrm{x}) \mathrm{dx}-\sum_{i=0}^{n} w_{i} f\left(x_{i}\right)
$$

\subsection{Definition}

An integration method of the form (3) is said to be of order $\mathrm{P}$, if it produces exact results $\left(\mathrm{E}_{\mathbf{n}}[\mathbf{f}]=0\right)$ for all polynomials of degree less than or equal to $\mathrm{P}[10]$.

In Open Newton-cotes rule, the end points of the interval is excluded in the function evaluation,i.e,

$$
\int_{\mathrm{a}}^{\mathrm{b}} \mathrm{f}(\mathrm{x}) \mathrm{dx}=\sum_{i=0}^{n} w_{i} f\left(x_{i}\right) \approx \int_{x_{-1}}^{x_{n+1}} f(x) d x
$$

for given $\mathrm{n}+1$ distinct points $\mathrm{x}_{0}<\mathrm{x}_{1}<\ldots<\mathrm{x}_{\mathrm{n}}$ and $\mathrm{n}+1$ weights $\mathrm{w}_{0}, \mathrm{w}_{1}, \ldots, \mathrm{w}_{\mathrm{n}}$ over the interval $(\mathrm{a}, \mathrm{b})$ with $\mathrm{x}_{\mathbf{i}}=\mathrm{a}+(\mathrm{i}+1) \mathrm{h}, \mathrm{i}=0,1,2, \ldots, \mathrm{n}$ and $h=\frac{b-a}{n+2}[7]$.

Therefore, the Open Newton cotes rules are given as follows,

If $\mathrm{n}=0$;

$$
\begin{aligned}
& \qquad \int_{\substack{\mathrm{a} \\
\text { where }} \in(a, b)}^{\mathrm{b}} \mathrm{f}(\mathrm{x}) \mathrm{dx}=(\mathrm{b}-\mathrm{a}) \mathrm{f}\left(\frac{\mathrm{a}+\mathrm{b}}{2}\right)+\frac{(b-a)^{3}}{24} f^{\prime \prime}(\xi), \\
& \text { If } \mathrm{n}=1 \text {; }
\end{aligned}
$$

$$
\begin{aligned}
\int_{a}^{b} f(x) d x=\frac{b-a}{2}\left[f\left(\frac{2 a+b}{3}\right)\right. & \left.+f\left(\frac{a+2 b}{3}\right)\right] \\
& +\frac{(b-a)^{3}}{36} f^{\prime \prime}(\xi)
\end{aligned}
$$


where $\xi \in(a, b)$.

If $\mathrm{n}=2$;

$$
\begin{aligned}
\int_{a}^{b} f(x) d x=\frac{b-a}{3} & {\left[2 f\left(\frac{3 a+b}{4}\right)-f\left(\frac{a+b}{2}\right)\right.} \\
+ & \left.2 f\left(\frac{a+3 b}{4}\right)\right] \\
+ & \frac{14}{45}\left(\frac{b-a}{4}\right)^{5} f^{(4)}(\xi),
\end{aligned}
$$

where $\xi \in(a, b)$.

$$
\text { If } \mathrm{n}=3 \text {; }
$$

$$
\begin{array}{r}
\int_{a}^{b} f(x) d x=\frac{b-a}{24}\left[11 f\left(\frac{4 a+b}{5}\right)+f\left(\frac{3 a+2 b}{5}\right)\right. \\
\left.+f\left(\frac{2 a+3 b}{5}\right)+11 f\left(\frac{a+4 b}{5}\right)\right] \\
+\frac{95}{144}\left(\frac{b-a}{5}\right)^{5} f^{(4)}(\xi),
\end{array}
$$

where $\xi \in(a, b)$.

In the closed Newton-cotes quadrature, the endpoints are included; whereas the open Newton- cotes quadrature, only the interior points are included. The corrected open Newton-cotes quadrature has higher precision than the classical quadrature rule. There are so many works has been done on the numerical improvement of Newtoncotes formulas.

Dehghan et al., presented an improvement of open, semi-open ,closed ,first and second kind [4 - 6, 8, 9] Chebyshev Newton- cotes quadrature rules. In the recent years, Clarence O.E Burg and his companions introduced a new family of derivative based rules $[2,3$, 7]. In 2013, Weijing Zhao and Hongxing Li [11] introduced a new family of closed Newton-cotes quadrature with Midpoint derivative rules.

In this paper, a new family of open Newton-cotes quadrature rule is described, which uses the derivative value at the Midpoint with their error terms. Also some numerical examples are given with their results and comparison. The result shows that the new formulas give better solution than the classical ones.

\section{2 .OPEN NEWTON - COTES \\ QUADRATURE WITH MIDPOINT DERIVATIVE}

A new Open Newton - Cotes Quadrature rules with Midpoint - Derivative is explained below which gives higher precision than the classical Newton-Cotes Quadrature rules.

\subsection{Theorem}

Open Newton - Cotes Quadrature with Midpoint Derivative for $(n=0)$ is

$$
\begin{aligned}
\int_{a}^{b} f(x) d x \approx(b-a) f\left(\frac{a+b}{2}\right) & \\
+ & \frac{(b-a)^{3}}{24} f^{\prime \prime}\left(\frac{a+b}{2}\right)
\end{aligned}
$$

The precision of this method is 3 .

Proof: Since the rule (9) has the degree of precision 1. Now we verify that the rule (9) is exact for

$$
\begin{aligned}
& \begin{array}{l}
\mathrm{f}(\mathrm{x})=\mathrm{x}^{2}, \mathrm{x}^{3} . \\
\text { When } f(x)=x^{2}, \int_{a}^{b} x^{2} d x=\frac{1}{3}\left(b^{3}-a^{3}\right)
\end{array} \\
& {[n=0] \Rightarrow(b-a)\left(\frac{a+b}{2}\right)^{2}+\frac{2(b-a)^{3}}{24}} \\
& \begin{array}{l}
\text { When } f(x)=x^{3}, \int_{a}^{b} x^{3} d x=\frac{1}{4}\left(b^{3}-a^{3}\right) . \\
{[n=0] \Rightarrow(b-a)\left(\frac{a+b}{2}\right)^{3}+\frac{6(b-a)^{3}}{24}\left(\frac{a+b}{2}\right)}
\end{array} \\
& \text { [n }=\frac{1}{4}\left(b^{4}-a^{4}\right) .
\end{aligned}
$$

Therefore, the precision of Open Newton - Cotes

Quadrature with Midpoint - Derivative is 3.

\subsection{Theorem}

Open Newton - Cotes Quadrature with Midpoint Derivative for $(n=1)$ is

$$
\begin{gathered}
\int_{\mathrm{a}}^{\mathrm{b}} \mathrm{f}(\mathrm{x}) \mathrm{dx} \approx \frac{\mathrm{b}-\mathrm{a}}{2}\left[\mathrm{f}\left(\frac{2 \mathrm{a}+\mathrm{b}}{3}\right)+\mathrm{f}\left(\frac{\mathrm{a}+2 \mathrm{~b}}{3}\right)\right] \\
+\frac{(b-a)^{3}}{36} f^{\prime \prime}\left(\frac{a+b}{2}\right),
\end{gathered}
$$

The precision of this method is 3 .

Proof: Since the rule (10) has the degree of precision 1.Now we verify that the rule (10) is exact for $\mathrm{f}(\mathrm{x})=$ $x^{2}, x^{3}$.

$$
\begin{aligned}
& \text { When } f(x)=x^{2}, \int_{a}^{b} x^{2} d x=\frac{1}{3}\left(b^{3}-a^{3}\right) \\
& \begin{aligned}
{[n=1] \Rightarrow\left(\frac{b-a}{2}\right)\left[\left(\frac{2 a+b}{3}\right)^{2}+\left(\frac{a+2 b}{3}\right)^{2}\right] } \\
+\frac{2(b-a)^{3}}{36}=\frac{1}{3}\left(b^{3}-a^{3}\right) .
\end{aligned}
\end{aligned}
$$




$$
\begin{aligned}
& \text { When } f(x)=x^{3}, \int_{a}^{b} x^{3} d x=\frac{1}{4}\left(b^{4}-a^{4}\right) \\
& \begin{aligned}
{[n=1] \Rightarrow\left(\frac{b-a}{2}\right) } & {\left[\left(\frac{2 a+b}{3}\right)^{3}+\left(\frac{a+2 b}{3}\right)^{3}\right] } \\
+ & \frac{6(b-a)^{3}}{36}\left(\frac{a+b}{2}\right)=\frac{1}{4}\left(b^{4}-a^{4}\right) .
\end{aligned}
\end{aligned}
$$

Therefore, the precision of Open Newton - Cotes Quadrature with Midpoint - Derivative is 3.

\subsection{Theorem}

Open Newton - Cotes Quadrature with Midpoint -

Derivative for $(n=2)$ is

$$
\begin{aligned}
\int_{a}^{b} f(x) d x \approx \frac{b-a}{3} & {\left[2 f\left(\frac{3 a+b}{4}\right)-f\left(\frac{a+b}{2}\right)\right.} \\
+ & \left.2 f\left(\frac{a+3 b}{4}\right)\right] \\
+ & \frac{14}{45}\left(\frac{b-a}{4}\right)^{5} f^{(4)}\left(\frac{a+b}{2}\right),
\end{aligned}
$$

The precision of this method is 5 .

Proof: Since the rule (11) has the degree of precision 3.Now we verify that the rule (11) is exact for

$$
\begin{aligned}
& f(x)=x^{4}, x^{5} \text {. } \\
& \text { When } f(x)=x^{4}, \int_{a}^{b} x^{4} d x=\frac{1}{5}\left(b^{5}-a^{5}\right) \text {; } \\
& {[n=2] \Rightarrow\left(\frac{b-a}{3}\right)^{a}\left[2\left(\frac{3 a+b}{4}\right)^{4}-\left(\frac{a+b}{2}\right)^{4}\right.} \\
& \left.+2\left(\frac{a+3 b}{4}\right)^{4}\right] \\
& +\frac{336}{45}\left(\frac{\mathrm{b}-\mathrm{a}}{4}\right)^{5}=\frac{1}{5}\left(b^{5}-a^{5}\right) \text {. } \\
& \text { When } f(x)=x^{5}, \int_{a}^{b} x^{5} d x=\frac{1}{6}\left(b^{6}-a^{6}\right) \text {; } \\
& {[n=2] \Rightarrow\left(\frac{b-a}{3}\right)\left[2\left(\frac{3 a+b}{4}\right)^{5}-\left(\frac{a+b}{2}\right)^{5}\right.} \\
& \left.+2\left(\frac{a+3 b}{4}\right)^{5}\right] \\
& +\frac{1680}{45}\left(\frac{\mathrm{b}-\mathrm{a}}{4}\right)^{5}\left(\frac{a+b}{2}\right)=\frac{1}{6}\left(b^{6}-a^{6}\right) \text {. }
\end{aligned}
$$

Therefore, the precision of Open Newton - Cotes Quadrature with Midpoint - Derivative is 5.

\subsection{Theorem}

Open Newton - Cotes Quadrature with Midpoint -

Derivative for $(n=3)$ i s

$$
\begin{aligned}
\int_{a}^{b} f(x) d x \approx \frac{b-a}{24} & {\left[11 f\left(\frac{4 a+b}{5}\right)+f\left(\frac{3 a+2 b}{5}\right)\right.} \\
+ & \left.f\left(\frac{2 a+3 b}{5}\right)+11 f\left(\frac{a+4 b}{5}\right)\right] \\
+ & \frac{95}{144}\left(\frac{b-a}{4}\right)^{5} f^{(4)}\left(\frac{a+b}{2}\right),
\end{aligned}
$$

The precision of this method is 5 .

Proof: Since the rule (12) has the degree of precision 3. Now we verify that the rule (12) is exact for $f(x)=$ $x^{4}, x^{5}$.

$$
\begin{aligned}
& \text { When } f(x)=x^{4}, \int_{a}^{b} x^{4} d x=\frac{1}{5}\left(b^{5}-a^{5}\right) \\
& \qquad \begin{aligned}
{[n=3] \Rightarrow\left(\frac{b-a}{24}\right) } & {\left[11\left(\frac{4 a+b}{5}\right)^{4}+\left(\frac{3 a+2 b}{5}\right)^{4}\right.} \\
+ & \left.\left(\frac{2 a+3 b}{5}\right)^{4}+11\left(\frac{a+4 b}{5}\right)^{4}\right] \\
+ & \frac{2280}{144}\left(\frac{\mathrm{b}-\mathrm{a}}{5}\right)^{5}=\frac{1}{5}\left(b^{5}-a^{5}\right)
\end{aligned}
\end{aligned}
$$

When $f(x)=x^{5}, \int_{a}^{b} x^{5} d x=\frac{1}{6}\left(b^{6}-a^{6}\right)$;

$$
\begin{aligned}
{[n=3] \Longrightarrow } & \left(\frac{b-a}{24}\right)\left[11\left(\frac{4 a+b}{5}\right)^{5}+\left(\frac{3 a+2 b}{5}\right)^{5}\right. \\
& \left.+\left(\frac{2 a+3 b}{5}\right)^{5}+11\left(\frac{a+4 b}{5}\right)^{5}\right] \\
+ & \frac{11400}{144}\left(\frac{\mathrm{b}-\mathrm{a}}{5}\right)^{5}\left(\frac{a+b}{2}\right)=\frac{1}{6}\left(b^{6}-a^{6}\right) .
\end{aligned}
$$

Therefore, the precision of Open Newton - Cotes Quadrature with Midpoint - Derivative is 5.

\section{THE ERROR TERMS OF OPEN NEWTON-COTES QUADRATURE WITH MIDPOINT DERIVATIVE}

The Error terms of Open Newton-Cotes Quadrature with Midpoint derivative are given below. The Error terms are the difference between the exact value and the quadrature rule.

\subsection{Theorem}

The Error term of Open Newton - Cotes Quadrature with Midpoint - Derivative for $(n=0)$ is

$$
\begin{gathered}
\int_{\mathrm{a}}^{\mathrm{b}} \mathrm{f}(\mathrm{x}) \mathrm{dx}=(\mathrm{b}-\mathrm{a}) \mathrm{f}\left(\frac{\mathrm{a}+\mathrm{b}}{2}\right)+\frac{(b-a)^{3}}{24} f^{\prime \prime}\left(\frac{a+b}{2}\right) \\
+\frac{(b-a)^{5}}{1920} f^{(4)}(\xi),
\end{gathered}
$$


where $\xi \in(a, b)$.This is fifth order accurate with the error term is

\section{Proof:}

$$
E_{1}[f]=\frac{(b-a)^{5}}{1920} f^{(4)}(\xi)
$$

$$
\begin{aligned}
& \text { Let } f(x)=\frac{x^{4}}{4 !}, \quad \frac{1}{4 !} \int_{a}^{b} x^{4} d x=\frac{1}{120}\left(b^{5}-a^{5}\right) ; \\
& (\mathrm{b}-\mathrm{a}) \mathrm{f}\left(\frac{\mathrm{a}+\mathrm{b}}{2}\right)+\frac{(b-a)^{3}}{24} f^{\prime \prime}\left(\frac{a+b}{2}\right) \\
& =\frac{b-a}{4 ! .16}\left(3 a^{4}+4 a^{3} b+2 a^{2} b^{2}+4 a b^{3}+3 b^{4}\right),
\end{aligned}
$$

Therefore,

$$
\begin{aligned}
\frac{1}{120}\left(b^{5}-a^{5}\right) & -\frac{b-a}{4 ! .16}\left(\begin{array}{c}
3 a^{4}+4 a^{3} b+2 a^{2} b^{2}+4 a b^{3} \\
+3 b^{4}
\end{array}\right) \\
& =\frac{(b-a)^{5}}{1920} .
\end{aligned}
$$

Therefore the Error term is,

$$
E_{1}[f]=\frac{(b-a)^{5}}{1920} f^{(4)}(\xi) .
$$

\subsection{Theorem}

The Error term of Open Newton - Cotes Quadrature with Midpoint - Derivative for $(n=1)$ is

$$
\begin{aligned}
& \int_{a}^{b} f(x) d x=\frac{b-a}{2}\left[f\left(\frac{2 a+b}{3}\right)+f\left(\frac{a+2 b}{3}\right)\right] \\
& +\frac{(b-a)^{3}}{36} f^{\prime \prime}\left(\frac{a+b}{2}\right)+\frac{19(b-a)^{5}}{38880} f^{(4)}(\xi),
\end{aligned}
$$

where $\xi \in(a, b)$.This is fifth order accurate with the error term is

$$
E_{2}[f]=\frac{19(b-a)^{5}}{38880} f^{(4)}(\xi) .
$$

\section{Proof:}

$$
\begin{aligned}
& \text { Let } f(x)=\frac{x^{4}}{4 !}, \quad \frac{1}{4 !} \int_{a}^{b} x^{4} d x=\frac{1}{120}\left(b^{5}-a^{5}\right) \\
& \frac{\mathrm{b}-\mathrm{a}}{2}\left[\mathrm{f}\left(\frac{2 \mathrm{a}+\mathrm{b}}{3}\right)+\mathrm{f}\left(\frac{\mathrm{a}+2 \mathrm{~b}}{3}\right)\right]+\frac{(b-a)^{3}}{36} f^{\prime \prime}\left(\frac{a+b}{2}\right) \\
& =\frac{b-a}{7776}\left(61 a^{4}+80 a^{3} b+42 a^{2} b^{2}+80 a b^{3}+61 b^{4}\right)
\end{aligned}
$$

Therefore,

$$
\begin{aligned}
\frac{1}{120}\left(b^{5}-a^{5}\right)- & \frac{b-a}{7776}\left(\begin{array}{c}
61 a^{4}+80 a^{3} b+42 a^{2} b^{2} \\
+80 a b^{3}+61 b^{4}
\end{array}\right) \\
& =\frac{19(b-a)^{5}}{38880} .
\end{aligned}
$$

Therefore the Error term is,

$$
E_{2}[f]=\frac{19(b-a)^{5}}{38880} f^{(4)}(\xi) .
$$

\subsection{Theorem}

The Error term of Open Newton - Cotes Quadrature with Midpoint - Derivative for $(n=2)$ is

$$
\begin{aligned}
& \int_{a}^{b} f(x) d x=\frac{b-a}{3}\left[2 f\left(\frac{3 a+b}{4}\right)-f\left(\frac{a+b}{2}\right)\right. \\
& \left.+2 f\left(\frac{a+3 b}{4}\right)\right] \\
& +\frac{14}{45}\left(\frac{b-a}{4}\right)^{5} f^{(4)}\left(\frac{a+b}{2}\right)+\frac{41(b-a)^{7}}{15482880} f^{(6)}(\xi),
\end{aligned}
$$

where $\xi \in(a, b)$.This is seventh order accurate with the error term is

$$
E_{3}[f]=\frac{41(b-a)^{7}}{15482880} f^{(6)}(\xi) .
$$

\section{Proof:}

$$
\begin{aligned}
& \text { Let } f(x)=\frac{x^{6}}{6 !}, \quad \frac{1}{6 !} \int_{a}^{b} x^{6} d x=\frac{1}{5040}\left(b^{7}-a^{7}\right) ; \\
& \begin{array}{r}
\frac{\mathrm{b}-\mathrm{a}}{3}\left[2 \mathrm{f}\left(\frac{3 \mathrm{a}+\mathrm{b}}{4}\right)-\mathrm{f}\left(\frac{\mathrm{a}+\mathrm{b}}{2}\right)+2 \mathrm{f}\left(\frac{\mathrm{a}+3 \mathrm{~b}}{4}\right)\right] \\
+\frac{14}{45}\left(\frac{\mathrm{b}-\mathrm{a}}{4}\right)^{5} f^{(4)}\left(\frac{a+b}{2}\right)
\end{array} \\
& =\frac{b-a}{3072.6 !}\left(\begin{array}{c}
4336 a^{6}+474 a^{5} b+351 a^{4} b^{2}+556 a^{3} b^{3} \\
+351 a^{2} b^{4}+474 a b^{5}+433 b^{6}
\end{array}\right) .
\end{aligned}
$$

Therefore,

$$
\begin{aligned}
& \frac{\left(b^{7}-a^{7}\right)}{5040} \\
& -\frac{b-a}{3072.6 !}\left(\begin{array}{c}
4336 a^{6}+474 a^{5} b+351 a^{4} b^{2}+556 a^{3} b^{3} \\
+351 a^{2} b^{4}+474 a b^{5}+433 b^{6}
\end{array}\right) \\
& =\frac{41(b-a)^{7}}{15482880}
\end{aligned}
$$$$
\text { Therefore the Error term is, }
$$

$$
E_{3}[f]=\frac{41(b-a)^{7}}{15482880} f^{(6)}(\xi) .
$$




\subsection{Theorem}

The Error term of Open Newton - Cotes Quadrature with Midpoint - Derivative for $(n=3)$ is

$$
\begin{aligned}
\int_{a}^{b} f(x) d x=\frac{b-a}{24} & {\left[11 f\left(\frac{4 a+b}{5}\right)+f\left(\frac{3 a+2 b}{5}\right)\right.} \\
+ & \left.f\left(\frac{2 a+3 b}{5}\right)+11 f\left(\frac{a+4 b}{5}\right)\right] \\
& +\frac{95}{144}\left(\frac{b-a}{5}\right)^{5} f^{(4)}\left(\frac{a+b}{2}\right) \\
+ & \frac{821(b-a)^{7}}{168.5^{5} \cdot 6 !} f^{(6)}(\xi), \quad, \quad(16)
\end{aligned}
$$

where $\xi \in(\mathrm{a}, \mathrm{b})$.This is seventh order accurate with the error term is

$$
E_{4}[f]=\frac{821(b-a)^{7}}{168 \cdot 5^{5} \cdot 6 !} f^{(6)}(\xi) .
$$

\section{Proof:}

$$
\text { Let } f(x)=\frac{x^{6}}{6 !}, \quad \frac{1}{6 !} \int_{a}^{b} x^{6} d x=\frac{1}{5040}\left(b^{7}-a^{7}\right) \text {; }
$$$$
\frac{b-a}{24}\left[11 f\left(\frac{4 a+b}{5}\right)+f\left(\frac{3 a+2 b}{5}\right)+f\left(\frac{2 a+3 b}{5}\right)\right.
$$$$
\left.+11 \mathrm{f}\left(\frac{\mathrm{a}+4 \mathrm{~b}}{5}\right)\right]
$$$$
+\frac{95}{144}\left(\frac{\mathrm{b}-\mathrm{a}}{5}\right)^{5} f^{(4)}\left(\frac{a+b}{2}\right)
$$

$=\frac{b-a}{375000.6 !}\left(\begin{array}{c}52985 a^{6}+57090 a^{5} b+44775 a^{4} b^{2}+65300 a^{3} b^{3} \\ +44775 a^{2} b^{4}+57090 a b^{5}+52985 b^{6}\end{array}\right)$.

Therefore,

$$
\begin{aligned}
& \frac{\left(b^{7}-a^{7}\right)}{5040} \\
& -\frac{b-a}{375000.6 !}\left(\begin{array}{c}
52985 a^{6}+57090 a^{5} b+44775 a^{4} b^{2}+65300 a^{3} b^{3} \\
+44775 a^{2} b^{4}+57090 a b^{5}+52985 b^{6}
\end{array}\right)
\end{aligned}
$$

Therefore the Error term is,

$$
=\frac{821(b-a)^{7}}{168 \cdot 5^{5} \cdot 6 !}
$$$$
E_{4}[f]=\frac{821(b-a)^{7}}{168 \cdot 5^{5} \cdot 6 !} f^{(6)}(\xi)
$$

\section{NUMERICAL RESULTS}

An approximate value of the following examples using the open Newton- cotes quadrature with Midpoint Derivative rules are determined and presented. To demonstrate the accuracy of the results, we evaluate the examples $1-4$ and the Comparison of results is shown in Tables $1-4$..
Table - 1: Exact value of $\int_{0}^{2} e^{x} \mathrm{dx}=6.3890560989$

\begin{tabular}{|c|c|c|c|c|}
\hline \multirow{2}{*}{$n$ value } & \multicolumn{2}{|c|}{ ONC } & \multicolumn{2}{c|}{ MDONC } \\
\cline { 2 - 5 } & App. value & Error & App. value & Error \\
\hline $\mathrm{n}=0$ & 5.436563657 & 0.952492441 & 6.342657600 & 0.046398498 \\
\hline $\mathrm{n}=1$ & 5.741401936 & 0.647654162 & 6.345464564 & 0.043515340 \\
\hline $\mathrm{n}=2$ & 6.361692569 & 0.027363529 & 6.388120309 & 0.000935789 \\
\hline $\mathrm{n}=3$ & 6.369923849 & 0.019132249 & 6.388287352 & 0.000768746 \\
\hline
\end{tabular}

Table - 2: Exact value of $\int_{0}^{2} \frac{\mathrm{dx}}{1+\mathrm{x}}=0.69314718$

\begin{tabular}{|c|c|c|c|c|}
\hline \multirow{2}{*}{$n$ value } & \multicolumn{2}{|c|}{ ONC } & \multicolumn{2}{c|}{ MDONC } \\
\cline { 2 - 5 } & App. value & Error & App. value & Error \\
\hline$n=0$ & 0.666666666 & 0.026480520 & 0.691358018 & 0.001789162 \\
\hline$n=1$ & 0.675000000 & 0.018147180 & 0.691460905 & 0.001686275 \\
\hline$n=2$ & 0.692063492 & 0.001083688 & 0.693023711 & 0.000123469 \\
\hline$n=3$ & 0.692377645 & 0.000769535 & 0.693044860 & 0.000102320 \\
\hline
\end{tabular}

Table -3 : Exact value of $\int_{0}^{1}\left(1+x^{4}\right) d x=1.2$

\begin{tabular}{|c|c|c|c|c|}
\hline \multirow{2}{*}{$n$ value } & \multicolumn{2}{|c|}{ ONC } & \multicolumn{2}{c|}{ MDONC } \\
\cline { 2 - 5 } & App. value & Error & App. value & Error \\
\hline$n=0$ & 1.06250000 & 0.13750000 & 1.187500000 & 0.012500000 \\
\hline$n=1$ & 1.104938272 & 0.095061728 & 1.188271605 & 0.011728395 \\
\hline$n=2$ & 1.192708333 & 0.007291666 & 1.200000000 & 0.000000000 \\
\hline$n=3$ & 1.194933333 & 0.005066666 & 1.200000000 & 0.000000000 \\
\hline
\end{tabular}

Table - 4: Exact value of $\int_{0}^{1} \mathrm{e}^{\mathrm{x}} \mathrm{dx}=6.361845641$

\begin{tabular}{|c|c|c|c|c|}
\hline \multirow{2}{*}{$n$ value } & \multicolumn{2}{|c|}{ ONC } & \multicolumn{2}{c|}{ MDONC } \\
\cline { 2 - 5 } & App. value & Error & App. value & Error \\
\hline $\mathrm{n}=0$ & 4.14816890 & 1.88015657 & 6.162322472 & 0.199523169 \\
\hline $\mathrm{n}=1$ & 5.05366896 & 1.30817667 & 6.174091231 & 0.187754410 \\
\hline $\mathrm{n}=2$ & 6.24259421 & 0.11925142 & 6.352885779 & 0.008959862 \\
\hline $\mathrm{n}=3$ & 6.27783380 & 0.08401183 & 6.354470691 & 0.007374950 \\
\hline
\end{tabular}

From the results presented in Tables 1 - 4, it is observed that the Open Newton-Cotes quadrature with midpoint derivative gives more accuracy than the classical ones. 


\section{CONCLUSION}

In this work we applied the Open Newton-Cotes Quadrature with Midpoint derivative over a finite interval $[\mathrm{a}, \mathrm{b}]$. The Error terms gives two orders of precision than the Standard methods.A numerical example is given to clarify the proposed Algorithm.

\section{REFERENCES}

[1] K.E.Atkinson, An Introduction to Numerical Analysis, John wiley and Sons , New York, NY, USA, Second Edition, 1989.

[2] Clarence O.E.Burg,"Derivative-based closed Newtoncotes numerical quadrature", Applied Mathematics and Computations, vol.218, pp. 7052-7065, 2012.

[3] Clarence O.E.Burg, and Ezechiel Degny, "Derivativebased midpoint quadrature rule, Applied Mathematics and Computations, vol.4, pp. 228-234, 2013.

[4] M.Dehghan, M.Masjed-Jamei, and M.R.Eslahchi, "On numerical improvementof closed Newton-Cotes quadrature rules", Applied Mathematics and Computations, vol.165, pp. 251-260, 2005.

[5] M.Dehghan, M.Masjed-Jamei, and M.R.Eslahchi, "The semi-open Newton-Cotes quadrature rule and its numerical improvement", Applied Mathematics and Computations, vol.171, pp. 1129-1140, 2005.

[6] M.Dehghan, M.Masjed-Jamei, and M.R.Eslahchi, "On numerical improvement of open Newton-Cotes quadrature rules", Applied Mathematics and Computations, vol.175, pp. 618-627, 2006.

[7] Fiza Zafar,Saira Saleem and Clarence O.E.Burg, "New Derivaive based open Newton- cotes quadrature rules ",Abstract and Applied Analysis, Volume 2014, Article ID 109138, 16 pages, 2014.

[8] S.M.Hashemiparast, M.R.Eslahchi,M.Dehghan, M.Masjed-Jamei,"On numerical improvement of the first kind Chebyshev-Newton-Cotes quadrature rules", Applied Mathematics and Computations, vol.174, pp. 1020-1032, 2006.

[9] S.M.Hashemiparast,M.Masjed-Jamei,

M.R.Eslahchi,M.Dehghan,"On numerical improvement of the second kind Chebyshev-Newton-Cotes quadrature rules(open type)", Applied Mathematics and Computations, vol.180, pp. 605-613, 2006.

[10]M.K.Jain,S.R.K.Iyengar and R.K.Jain, Numerical methods for Scientific and Computation, New Age International (P) limited, Fifth Edition ,2007.

[11] Weijing Zhao and Hongxing,"Midpoint Derivative-Based Closed Newton- Cotes Quadrature", Abstract and Applied Analysis, vol.2013, Article ID 492507,10 pages, 2013 